\title{
Site-Sensitive Gas Sensing and Analyte Discrimination in Langmuir-Blodgett Porphyrin Films
}

\author{
G. Bussetti, A. Violante, R. Yivlialin, S. Cirilli, B. Bonanni, P. Chiaradia, and C. Goletti* \\ Dipartimento di Fisica, Università di Roma “Tor Vergata”, Via della Ricerca Scientifica, 00133 Roma, Italy
}

\section{Tortora and R. Paolesse}

Dipartimento di Scienze e Tecnologie Chimiche, Università di Roma “Tor Vergata”, Via della Ricerca Scientifica, 00133 Roma, Italy

\author{
E. Martinelli, A. D’Amico, and C. Di Natale \\ Dipartimento di Ingegneria Elettronica, Università di Roma “Tor Vergata”, Via del Politecnico, 00133 Roma, Italy
}

\author{
G. Giancane and L. Valli \\ Dipartimento di Ingegneria dell’Innovazione, Università del Salento, Via Monteroni, 73100 Lecce, Italy
}

ABSTRACT: We have studied the controlled adsorption of molecules of volatile compounds on thin Langmuir-Blodgett films of porphyrins deposited onto the surface of a quartz microbalance. The modification of the film optical anisotropy has been measured by reflectance anisotropy spectroscopy (RAS), and simultaneously, the amount of adsorbed molecules has been monitored. Optical spectra measured after adsorption of single analytes are interpreted straightforwardly in terms of anisotropies related to the $\mathrm{H}$-like and J-like aggregation modes of porphyrins. By an appropriate selection of analytes, we have then investigated the effect of

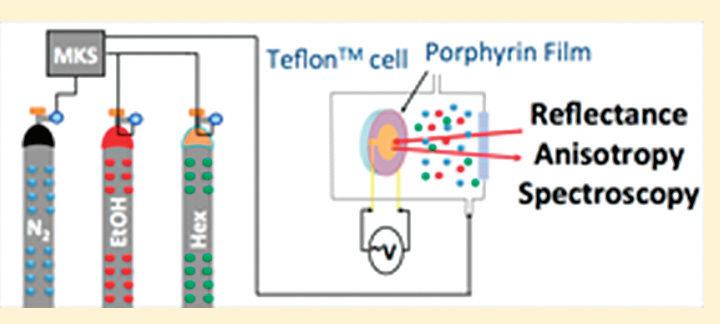
the exposure to controlled vapor mixtures. The experimental RAS line shape variation with respect to the signal measured before exposure is the sum of the variations produced by single analytes. This shows that the identification of types and even quantities of gases and vapors in gas sensing experiments is possible, and the responses of the sensing layer to single analytes could be listed in a library.

\section{INTRODUCTION}

Chemical sensors are logically (and in many cases also practically) split in two main components: the sensing layer and the transducer device, necessary to convert the presence of molecules in the environment into a detectable signal. On the sensing layer the vapor molecules are either adsorbed or undergo a chemical transformation, via processes producing the variation of a physical quantity, which is measured by a suitable method, usually through the conversion into an electric signal. ${ }^{1}$

In some cases, the measurable quantity is a property of the adsorbed molecules; in other cases, it is a characteristic of the adsorbing layer. For instance, mass sensitive devices such as a quartz microbalance measure a property of the adsorbate (the total adsorbed mass). ${ }^{2}$ On the other hand, an optical transducer typically measures changes in the optical property of the adsorbing material. ${ }^{3}$ Two sensors can then exhibit different behaviors, even if they are based on the same sensing material.

At the current state of the art, all sensors measure the property of large collections of interacting units: except for some sophisticated technique that requires particular operating conditions, such as low temperature or very low pressure, single adsorption events cannot be measured. Minimum readable signals are then obtained by a multitude of individual sensing events: in a typical quartz microbalance about 1 pmol of adsorbed molecule is necessary to get a measurable frequency shift (typically about $1 \mathrm{~Hz}$ change with respect to a $20 \mathrm{MHz}$ signal frequency).

This produces a hiatus between the chemical design of sensing units and the effective use in a device. The match between the two realms is provided by the molecular film technology, which offers the practice to construct an ensemble of the sensing units necessary for the real device. ${ }^{4}$ In the past few years, this technology has been complemented by the principles of supramolecular chemistry, exploiting the sensing properties of molecular assemblies kept together by noncovalent forces. ${ }^{5}$ It has been demonstrated that in many cases the sensing properties of these assemblies may largely exceed those of the individual constituents. ${ }^{6}$ Such arrangements can also involve large highly oriented areas, as it happens in Langmuir-Blodgett (LB) films.

\footnotetext{
Received: January 11, 2011

Revised: March 16, 2011

Published: April 04, 2011
} 
A significant example is offered by metalloporphyrin based sensors, where the property of the individual porphyrin units are still found in the behavior of sensors coated by a large amount of such molecules, but additional properties emerge when supramolecular porphyrin ensembles are considered. ${ }^{7}$

For the particular structure of porphyrins, the simultaneous presence of various interaction mechanisms (in particular axial coordination, $\pi-\pi, \pi$-cation, and hydrogen bond interactions) is expected to take place between individual porphyrins and guest molecules. ${ }^{8}$ In addition, unavoidable van der Waals forces should be considered, whose importance is amplified in molecular films. ${ }^{9}$

Recently we have studied supramolecular assemblies of metalloporphyrins, in particular by investigating the sensitivity of the optical properties of porphyrin films exposed to volatile molecules. In a previous paper ${ }^{10}$ we have shown that ethanol, hexane, and trimethylamine produce different and distinguishable modifications in the optical anisotropy of Langmuir-Schaeffer (LS) films of a Zn-porphyrin. As a result, optical anisotropy can enable the identification of classes of molecules on the basis of the interaction involved in the adsorption process.

LS systems offer in general a large surface-to-volume ratio, meaning a wide sensing area for adsorbing volatile molecules. However, the higher structural order of LB layers is expected to provide a larger anisotropy signal, that is, a higher sensitivity to the adsorption process monitored by reflectance anisotropy spectroscopy (RAS). ${ }^{11}$ We have therefore performed new experiments on a LB thin film of the same porphyrin, to exploit the more ordered arrangement of the molecules due to this deposition technique.

We have measured the optical anisotropy modification of an 8 monolayer LB thin film of a 5,10,15,20-tetrakis(4-heptyloxyphenyl)porphyrin (Zn-HepOTTP), due to the exposure to three different volatile organic compounds (VOCs). The molecular film was deposited onto the surface of a quartz microbalance (QMB) providing a measure of the amount of adsorbed molecules. In this paper we have exploited only the qualitative information drawn from QMB data: a quantitative comparison between QMB and optical anisotropy will be reported elsewhere. RAS spectra are demonstrated to be the signature of the adsorption of single analytes on the film. As a consequence, in the anisotropy signal read-out after exposure to mixture of analytes, the contributions of single analytes can be singled out.

\section{EXPERIMENTAL SECTION}

Zn-HepOTTP, prepared from 4-heptyloxybenzaldehyde and pyrrole by the Adler method, ${ }^{12}$ has been dispersed onto pure water from chloroform spreading solutions (concentration $4 \times$ $10^{-4} \mathrm{M}$ ). Langmuir depositions were carried out by a KSV 5000 system $3 \mathrm{LB}$ apparatus $\left(850 \mathrm{~cm}^{2}\right)$. Ultrapure water (Millipore Milli-Q, 18.2 M $\Omega \mathrm{cm}$ ) was used as the subphase. The temperature of the subphase was regulated at $20^{\circ} \mathrm{C}$ by a Haake GH-D 8 apparatus. The floating film has been compressed by means of two Teflon barriers, and the Langmuir curve (surface pressure vs area per molecule) has been acquired. The film formed at the air-water interface has been transferred onto a hydrophobic substrate by LB technique (vertical dipping, speed of $5 \mathrm{~mm} / \mathrm{min}$ during both upstroke and downstroke) at a surface pressure of $25 \mathrm{mN} / \mathrm{m}$, that corresponds to the maximum molecular package.

The film (nominal thickness 8 monolayers) was deposited on both surfaces of a piezoelectric quartz crystal, with a gold-coated central circular area (diameter $4 \mathrm{~mm}$ ) for the QMB electrodes. The quartz surface was properly polished to obtain a high-quality

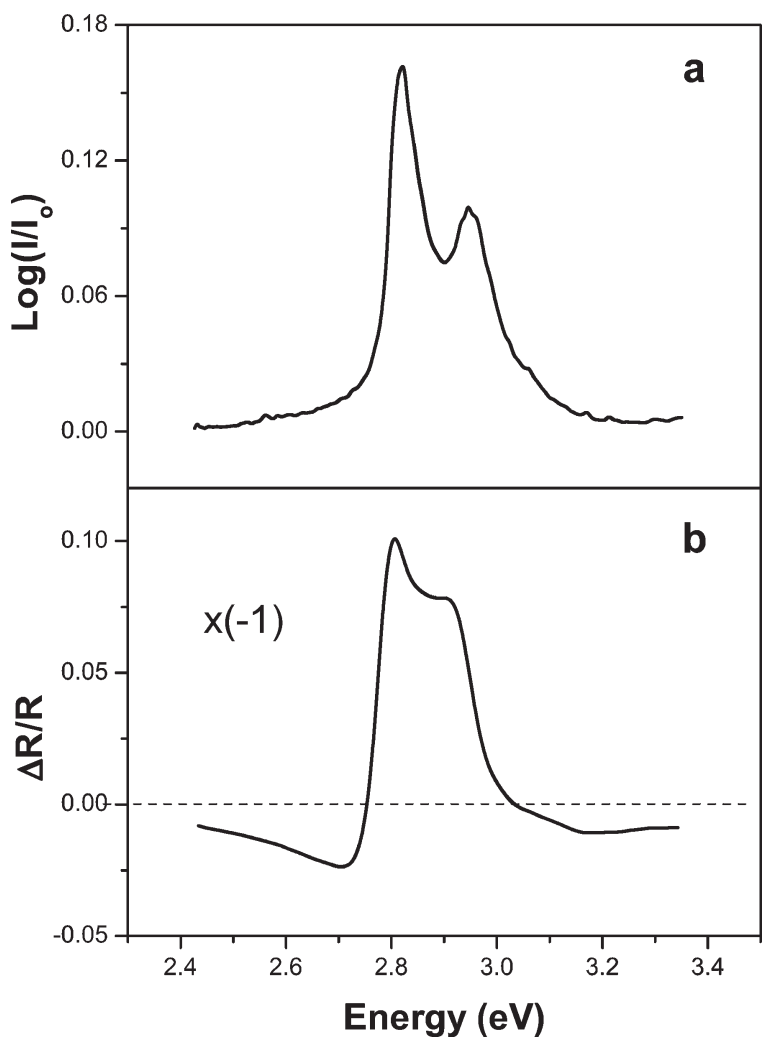

Figure 1. (a) Absorption spectrum of an 8 MLs thick Zn-HepOTTP Langmuir-Blodgett film. The two structures at $2.80 \pm 0.02 \mathrm{eV}$ and 2.92 $\pm 0.02 \mathrm{eV}$ are related to, respectively, J-like and $\mathrm{H}$-like aggregation of the porphyrins in the layer. (b) $\Delta R / R$ spectrum of the same porphyirn layer before the exposure to VOCs. The sign of the RAS signal (arbitrary) has been chosen here to compare spectra reported in two panels. As a result, this RAS spectrum has been multiplied by a factor of -1 with respect to spectra reported in further figures.

light reflectance. In the absorbance spectrum (Figure 1, panel a) the typical signature of $\mathrm{Zn}$-HepOTTP layers is observed, as reported in literature. ${ }^{13}$

Details for the QMB apparatus, measuring the frequency shift of an oscillator circuit, linearly related to the adsorbed mass according to the Sauerbrey equation, have been reported in ref 10. The QMB was placed in a Teflon cell endowed with inlet and outlet gates for gases. A glass viewport allowed the optical access to the sample (the optical signal due to the window birefringence is usually negligible, and in principle can be subtracted).

In a RAS experiment, linearly polarized light, alternatively directed along two orthogonal directions $(\alpha$ and $\beta$ ) via a photoelastic-modulator ${ }^{14}$, is shone on the sample at near normal incidence, and the reflected beam is collected in the detector. In the experiment reported here, we carefully focused the light beam onto a zone of the quartz substrate not gold coated, to exploit the role of the substrate in the signal and then gain a simple interpretation of the data (as explained below).

The ratio between $\Delta R$ (the difference of differently polarized reflected light) and the average reflectivity $R$ expresses the result of the measurement at a certain photon energy, that is, the anisotropy of the sample reflectance between directions $\alpha$ and $\beta$ :

$$
\frac{\Delta R}{R}=2 \times \frac{R_{\alpha}-R_{\beta}}{R_{\alpha}+R_{\beta}}
$$


$R_{\alpha}\left(R_{\beta}\right)$ is the intensity of light reflected from the sample for light polarized along $\alpha(\beta)$. In our experiment, the sample has been oriented by measuring the dependence of RAS spectra upon the azimuthal rotation, and selecting the position that maximizes the signal. ${ }^{15} \Delta R / R$ spectra presented in this paper have been measured in the vis-near-UV range $(2.5-3.4 \mathrm{eV})$ where the main absorption structure of the porphyrin (Soret band) is found. The whole apparatus and most significant applications on organics have been reported elsewhere. ${ }^{16,17}$

$\Delta R / R$ signal, within the three-layer model,${ }^{18}$ is expressed as

$$
\Delta R / R=\mathrm{d}\left[A \Delta \varepsilon_{1}^{\prime \prime}-B \Delta \varepsilon_{1}^{\prime}\right]
$$

where $\Delta \varepsilon_{1}^{\prime}$ and $\Delta \varepsilon^{\prime \prime}{ }_{1}$ are, respectively, the real and the imaginary parts of the dielectric function anisotropy of the layer, $d$ is the layer thickness, and $A$ and $B$ express the dependence upon the substrate optical properties (for definition of $A$ and $B$, see ref 19).

RAS and QMB signals have been measured simultaneously to correlate the uptake of VOCs with the variation of the optical anisotropy of the sensor. ${ }^{10}$ Each analyte can interact with the sensing layer according to various processes, depending on both selective mechanisms, due to particular chemical functionalities present in the molecule, and on nonselective dispersion interactions. To check the effect of different interactions the organic surface was exposed to three typical molecules representative of distinct families, alcohols (ethanol), amines (butylamine), and alkanes (hexane), which define a well-assorted blend of van der Waals, hydrogen bond, and coordination interactions.

At standard temperature and pressure, these are liquid analytes: their saturated vapors (obtained by $\mathrm{N}_{2}$ bubbling at constant temperature) were diluted in a pure nitrogen carrier and injected into the measurement cell. Saturated vapor concentrations were calculated by the empirical Antoine's law. ${ }^{20}$ Dilution ratios for each VOC as well as defined mixtures of different VOCs were accurately regulated by a set of mass flow controllers (MKS Instruments). In each measurement the porphyrin film was exposed to vapor(s) for about $3 \mathrm{~min}$ (necessary to reach a steady state for the QMB signal); then, after the measurements it was flushed with a pure nitrogen flow for about $20 \mathrm{~min}$ to restore the initial conditions (cleaning). All measurements were performed at room temperature in a thermally conditioned laboratory.

\section{RESULTS AND DISCUSSION}

The optical anisotropy of the as grown LB film was almost insensitive to analytes: despite the evident variation of the simultaneously monitored QMB signal (demonstrating the adsorption of VOC molecules on the film), RAS measured a nearly constant signal during exposure. All the reported spectra have then been recorded after a preliminary exposure of the as grown film to saturated vapors of ethanol diluted with $\mathrm{N}_{2}$ carrier gas (70\%). After cleaning this activated sample in nitrogen flow, the optical anisotropy (previously inactive) of the LB film exhibited now a high sensitivity to volatile compounds introduced in the cell, comparable to the published LS film case. ${ }^{10} \mathrm{On}$ the contrary, the adsorption isotherms recorded by QMB before and after activation (not reported here) are identical: further adsorption processes are then unaffected by activation.

This situation could evoke a case reported very recently in the literature: ${ }^{21}$ some organic films exhibit a different, higher degree of order after the exposure to particular chemical solvents, via a reorganization of the material in the outer layer ascribed to a "solvent annealing" effect. However, this explanation is less satisfactory in our case: ethanol is not a solvent for $\mathrm{Zn}$ HepOTTP, and consequently, it is not likely that a reorganization of the supramolecular structures of the LB film takes place upon the very high first exposure to ethanol. The experiments show that the treatment does not modify the RAS spectrum: this implies that the arrangement of neighboring molecules, determining the optical anisotropy signal in LB and in LS films, ${ }^{10,15}$ is practically unaltered. Nevertheless, a careful AFM (Atomic Force Microscope) investigation of the film after and before activation will be carried out, to establish if long-range as well as short-range modifications happen in the film after the exposure to ethanol. Also, a pre-existent contamination of the as grown film due to impurities coming from the preparation procedure will be examined by exposing to analytes porphyrin films of different thickness deposited in vacuum. The activation in this case would mean a sort of cleaning able to clear the adsorption sites available on the film. New experiments are in preparation to understand the role of ethanol and explain the high concentration value necessary to cause the effect.

The RAS spectrum for the activated LB film (before exposure to analytes) is reported in Figure 1b. It has a different line shape with respect to the corresponding spectrum of a thicker LS sample of the same porphyrin (see Figure 1 of ref 10). This result is not surprising: the different film thickness ( 30 MLs vs 8 MLs) implies a likely different arrangement of the porphyrin molecules in the sample and, consequently, a different optical anisotropy. Moreover, for the high thickness value of the LS film, a different interpretation of the reflected intensity should be adopted, as the assumption of the three-layer model (layer thickness $\ll \lambda$ ) is no longer satisfied. ${ }^{18}$

Since the quartz substrate is transparent $(B=0)$ in the whole photon energy range, ${ }^{19}$ then $\Delta R / R$ correctly mimics the absorption anisotropy spectrum (see eq 2): the RAS spectrum in Figure $1 \mathrm{~b}$ is similar to the corresponding absorption spectrum measured with unpolarized light by a spectrophotometer on the same sample (Figure 1a). It is known that the absorption spectrum of an organic layer is a reliable tool to control the aggregation of the molecules in the solid state: spectral components differently shifted with respect to the bands of individual units measured in solution are related to molecular aggregation, specifically $\mathrm{J}$ and $\mathrm{H}$ aggregations. ${ }^{22}$ In the former, two adjacent porphyrins are stacked with a certain offset (edge-to-edge configuration; the related component in panel $\mathrm{b}$ of Figure 1 is redshifted with respect to the Soret band of the solution); in the latter two adjacent porphyrins are parallel (face-to-face configuration, whose spectral component in panel b of Figure 1 is blueshifted with respect to the porphyrin solution Soret band). It should be noted that $\mathrm{J}$ aggregation is usually predominant in a porphyrin layer. ${ }^{10}$

The similarity of RAS and absorption spectra suggests taking advantage of the high sensitivity of RAS (up to $10^{-5}$ of the optical signal) to detect the slightest signal variations due to modifications in the uppermost region of the LB system, ${ }^{23}$ in particular to identify straightforwardly the contributions related to J-like and $\mathrm{H}$-like aggregation in the layer (respectively at $2.80 \pm 0.02 \mathrm{eV}$ and at $2.92 \pm 0.02 \mathrm{eV}$ ). This means that if we monitor at characteristic photon energies the modification of the anisotropy signal brought about by the exposure to analytes, we could extract the information of which porphyrin configuration is better suited for the adsorption of a certain vapor molecule. In other words, we could determine that a VOC preferentially adsorbs onto H-like or J-like aggregation sites of the film. In this sense, the 


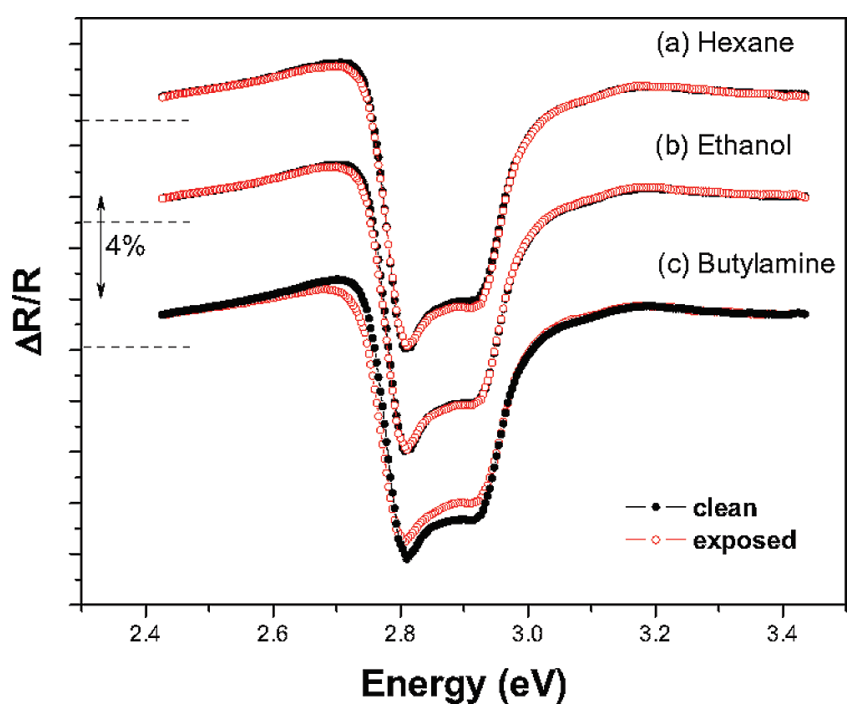

Figure 2. $\Delta R / R$ spectra measured on a $8 \mathrm{ML}$ thick Zn-HepOTTP Langmuir-Blodgett film: (a) before $(-, 0)$ and after $(---, \bigcirc)$ the exposure to hexane vapors $(18 \%)$; (b) before $(-, 0)$ and after $(---, \bigcirc)$ the exposure to ethanol vapors $(18 \%)$; (c) before $(-, 0)$ and after $(--,, \bigcirc)$ the exposure to butylamine (1\%). Each exposure stage was $210 \mathrm{~s}$ long. The sign of $\Delta R / R$ has been chosen arbitrarily. Zero-lines have been reported for each curve.

information delivered from RAS, although averaged in the macroscopic region covered by the light spot, allows an insight in the microscopic structure of the system: if a particular spectral feature, associated to particular sites or bonds at the layer, disappears or modifies after the organic layer has been exposed to a vapor, the preferential adsorption of the analyte to that site or bond can be reasonably inferred. ${ }^{24}$

In Figure 2 we compare the RAS spectra for the 8 MLs thick LB film before and after the activated sample has been exposed to (respectively) hexane (18\%, corresponding to $27180 \mathrm{ppm}$, curve a), ethanol (18\%, corresponding to $10656 \mathrm{ppm}$, curve b), and butylamine (1\%, $1475 \mathrm{ppm}$, curve c) vapors. These dilution values (corresponding to different concentrations) have been chosen to gain a well detectable RAS signal level: for butylamine a low concentration has been used, because this analyte strongly interacts with the porphyrin, producing sometime irreversible modifications in the spectra. Reversibility of the adsorption process is observed for ethanol and hexane: after cleaning in nitrogen flux, the LB film recovers the same optical anisotropy spectrum (the same happens for the QMB signal). Longer times are necessary to recover the initial spectrum as well as the QMB signal intensity in the case of butylamine adsorption, even at low dilution values.

The variation produced by adsorption is highlighted by drawing the difference between RAS spectra measured after and before exposure: $\triangle \mathrm{RAS}=\Delta R / R_{\mathrm{after}}-\Delta R / R_{\text {before }}$. The effect of adsorption for the three cases is reported in Figure 3. The line shape of hexane and ethanol displays a certain similarity: for the reported sign of $\Delta R / R$ (arbitrary choice), we observe a sharp minimum at about $2.75 \mathrm{eV}$, then a maximum at about $2.82 \mathrm{eV}$, followed by a further broad minimum between 2.85 and $3.00 \mathrm{eV}$. The two structures at higher photon energies are nearly coincident with $\Delta R / R$ features previously related to, respectively, J-like $(2.80 \mathrm{eV})$ and H-like $(2.92 \mathrm{eV})$ aggregation. Given the sign of $\triangle R A S$, in our signal a positive value means that the

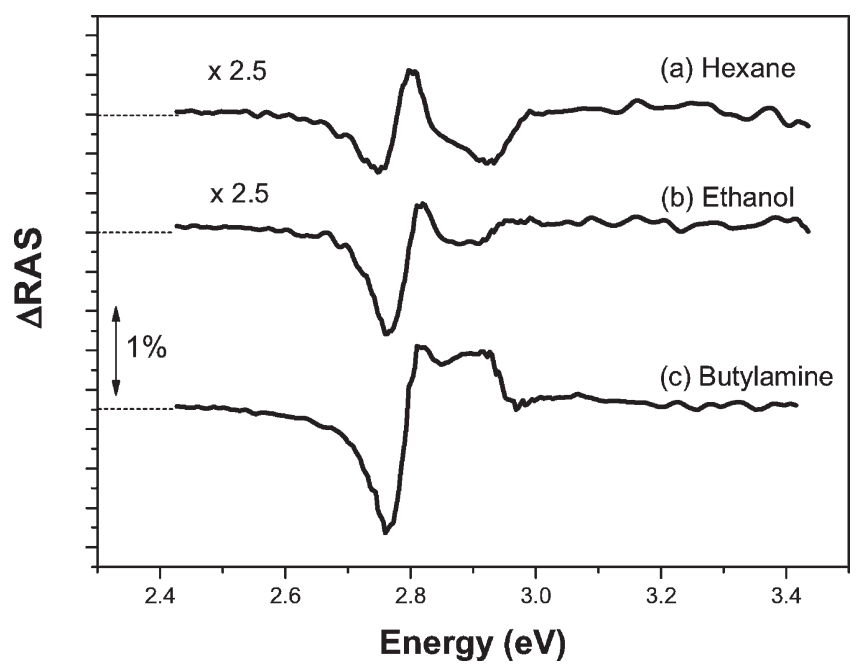

Figure 3. $\triangle R A S$, defined as the difference $\Delta R / R_{\text {after }}-\Delta R / R_{\text {before }}$ between RAS spectra (reported in Figure 1) measured before $(\Delta R /$ $\left.R_{\text {before }}\right)$ and after $\left(\Delta R / R_{\text {after }}\right)$ the exposure of a $8 \mathrm{ML}$ thick $\mathrm{Zn}$ HepOTTP Langmuir-Blodgett film to (a) hexane (18\%); (b) ethanol (18\%); (c) butylamine (1\%). $\triangle$ RAS values for hexane and ethanol have been multiplied by a factor of 2.5. Zero-lines have been reported in all cases.

corresponding anisotropy has decreased after exposure: this is consistent with a preferential interaction of volatile molecules on $\mathrm{J}$ aggregates (and consequently a lower number of unaffected $\mathrm{J}$ aggregates) or a larger disorder. A negative value means that the corresponding anisotropy has increased, as if more $\mathrm{H}$ aggregates produce the signal from the film. The complexity of the system suggests some mechanisms that are able to explain this result: the modification of the structure of the film (swelling ${ }^{25}$ ) due to the volatile molecules adsorption can induce porphyrin molecules to assume $\mathrm{H}$ aggregation geometry, or the relative arrangement of layers (giving $\mathrm{H}$-like aggregation because of the stacking) is modified. In the energy region below $2.80 \mathrm{eV}$, the effect of the change of broadening of the different spectral components very likely due to the disorder induced by adsorption cannot be disentangled.

The particular structural complexity of the LB layer, and a certain sample dependence of RAS line shape, hampers a more detailed description of the analyte-porphyrin interaction, but a general overview plus a methodology of investigation can be inferred: a more precise investigation will become possible when a controlled, homogeneous, well ordered organic system (possibly a single layer) will be studied, although it is important to note that a single monolayer is of low interest for sensing purpose, due to the corresponding low saturation limit. Nevertheless, new experiments on one porphyrin monolayer evaporated in ultra high vacuum on solid single crystal surfaces are in progress to help the interpretation of this scientific issue.

If we take into account the different concentration value and normalize the measured RAS variation with respect to the number of analyte molecules, we obtain that ethanol is more reactive than hexane: about a factor of 3 fewer molecules are necessary for producing the same $\triangle \mathrm{RAS}$ amplitude. This finding is expected, as ethanol interacts with porphyrins via dispersion forces, plus a (weak) coordination interaction with the inner core $\mathrm{Zn}$ ion and hydrogen bond with peripheral ether groups, these latter terms exceeding the simple dispersion force term of hexane. 


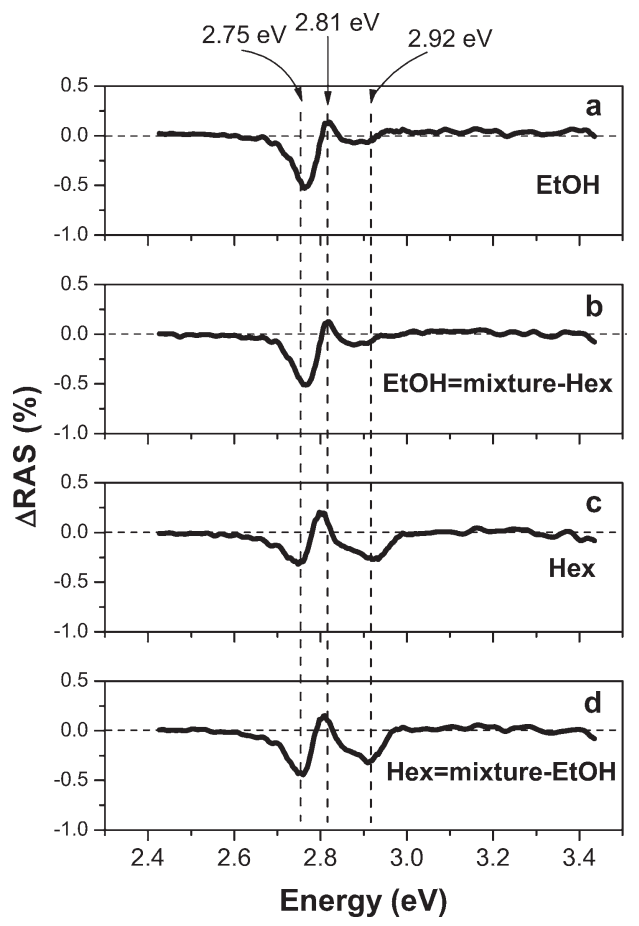

Figure 4. $\triangle$ RAS spectra measured (d) and computed (c) for the exposure of the LB film to a mixture of analytes: A + B (A, ethanol, panel a; B, hexane, panel $b$ ). The dilution value for both ethanol and hexane was $18 \%$. Zero-lines have been reported in all cases.

Also, the higher effect of amine witnessed by RAS is consistent with the strength of its reactivity with porphyrins. In this case the high affinity of $\mathrm{Zn}$ porphyrin toward amine coordination induces significant structural modifications, where both $\mathrm{H}$-like and J-like aggregation are clearly affected.

The spectra reported in Figure 3 demonstrate that, in the considered cases, the different interactions between analyte and porphyrin result in characteristic anisotropy modifications. The optical signature of the interaction is helpful when the film is exposed to controlled mixtures of analytes. In particular, the LB film has been exposed to known mixtures of two analytes (hereafter indicated as A and B) in two distinct experiments: (1) in the former, analytes $\mathrm{A}$ and $\mathrm{B}$ were introduced simultaneously in the cell (mixture A + B, spectra reported in Figure 4); (2) in the latter, at a certain time the inlet of one of two analytes has been interrupted, then exposing the sample only to the residual analyte (spectra reported in Figure 5).

The signal measured during exposure to the mixture $A+B$ (Figure $4 \mathrm{~d}$ ) is nearly coincident with the computed $\triangle \mathrm{RAS}$ (Figure 4c) obtained by summing the single experimental $\triangle \mathrm{RAS}$ measured separately when the film has been exposed only to A or $B$ (at the same concentration value) (Figure $4 a, b)$. Then, subtracting $\triangle \mathrm{RAS}_{\mathrm{A}}$ (for analyte $\mathrm{A}$ ) from $\triangle \mathrm{RAS}_{\mathrm{A}+\mathrm{B}}$ (for mixture $\mathrm{A}+\mathrm{B}$ ) one obtains exactly the signal measured for $\mathrm{B}$ only $\left(\triangle \mathrm{RAS}_{\mathrm{B}}\right)($ Figure 5$)$. The same happens for the other analyte $\mathrm{B}$, as we demonstrate for ethanol (Figure $5 b$ ) or for hexane (Figure $5 \mathrm{~d}$ ). We define this commutative behavior as "linear". This is not apparent when butylamine enters the mixture (data not reported), as it introduces a nonlinear behavior. The coordination binding of butylamine to the porphyrin film can be confidently considered the reason of the different influence exhibited by this analyte. This strong interaction in fact allows butylamine to displace

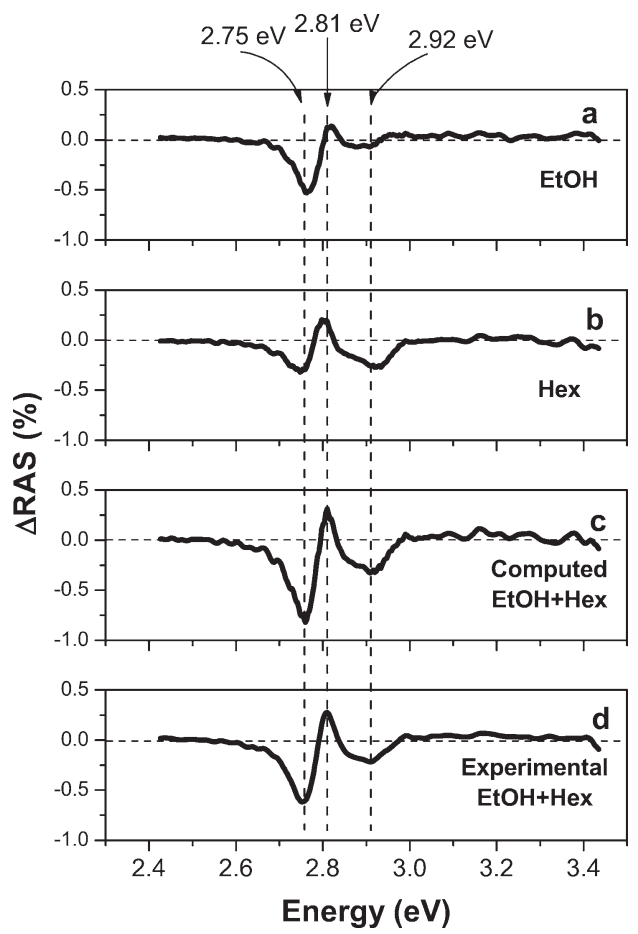

Figure 5. $\triangle$ RAS spectra measured for the exposure of the LB film to a mixture of analytes A $+B$ after the inlet of single analytes (A or B) has been separately interrupted (panel $b$, after hexane inlet interruption; panel d, after ethanol inlet interruption). The RAS spectra of analyte A (ethanol) and analyte B (hexane) are reported, respectively, in panels a and $\mathrm{c}$. The dilution value for both ethanol and hexane was $18 \%$. Zerolines have been reported in all cases.

both ethanol and hexane from the porphyrin binding sites, resulting in the nonlinear behavior of $\triangle \mathrm{RAS}$ spectra variations when the film is exposed to butylamine containing mixtures.

The consequence is significant for sensing applications: measuring the signal of the film for known concentrations of single analytes, the optical anisotropy variations for VOCs could be listed in a sort of analyte library, to deconvolve into single components the complex RAS spectrum measured after the sensor has been exposed to an unknown mixture of those VOCs. More properly, this finding suggests the possibility to discriminate among volatile compounds according to the interaction mechanisms that binds the molecule to the porphyrin layer. In the case illustrated here for each molecule a dominant interaction mechanism can be found: dispersion interaction for hexane and coordination for ethanol. It is important to remark that in many other volatile compounds more than one interaction mechanism can coexist: the study of the RAS signal in these situations will be the subject of successive investigations.

The development of optical sensors for the detection of gaseous analytes is strongly motivated by the need for economical, accurate, and reliable devices. The alteration of porphyrin colors upon exposure to volatile compounds is a well-known process. Porphyrin LB films have been recently used to monitor VOCs strongly interacting with the porphyrin molecules, able to produce an evident modification of the optical absorption spectrum visible even by the naked eye (colorimetric gas detectors). ${ }^{26}$ The detection of ethanol at concentrations below $100 \mathrm{ppm}$ has been achieved by a porphyrin coated photodetector illuminated by a blue-light LED. ${ }^{27}$ It has to be also remarked that 
adsorption of hexane can be hardly detected because it does not influence directly porphyrin optical spectra other than to induce the solid film swelling.

Although at this stage the development of a sensor would be premature, the RAS study reported here indicates a strategy for the recognition of an analyte that interacts even weakly with the sensitive film, exploiting the extremely high sensitivity of the technique. RAS is demonstrated to be able to discriminate the different interactions taking place in an ordered layer of hostguest systems, exceeding the sensitivity limit of other commonly used optical methods. The specific response to single analytes shows that the identification of vapors interacting with the sensing layer is a possible task via an appropriate analysis of RAS data: the separation into individual contributions, each related to a different interaction mechanism, can be achieved by an appropriate multivariate data analysis. ${ }^{28}$ It has to be mentioned that deviations from simple linear superpositions of single effects are observed for strongly interacting compounds, such as butylamine for a porphyrins film.

\section{CONCLUSIONS}

In this paper, we have reported how, by monitoring the exposure of a LB porphyrin film to volatile compounds by reflectance anisotropy spectroscopy, we gain mechanistic information about the adsorption process onto the film. In particular, despite the complexity inherent to the LB deposition process, we singled out the adsorption of the investigated vapors on $\mathrm{H}$ or $\mathrm{J}$ aggregation modes of the porphyrin in the film. The exposure of the organic film to controlled mixtures of vapors demonstrates that the effects of different analytes (not strongly interacting with porphyrins) combine linearly in the resulting experimental RAS signal. This paves the way to the possibility of single analyte recognition by an appropriate deconvolution of the experimental anisotropy readout.

\section{AUTHOR INFORMATION}

\section{Corresponding Author}

*E-mail: goletti@roma2.infn.it.

\section{ACKNOWLEDGMENT}

The authors wish to thank Mr. Luigi Dimo for technical assistance during the whole Langmuir depositions.

\section{REFERENCES}

(1) Grundler, P. Chemical Sensors; Springer Verlag AG: Berlin, 2007.

(2) Ballantine, D. S.; White, R. M.; Martin, S. J.; Ricco, A. J.; Zellers, E. T.; Frye, G. C.; Wohltjen, H. Acoustic Wave Sensors; Academic Press: San Diego, CA, 1997.

(3) Wolfbeis, O. S. Adv. Mater. 2008, 20, 3759-3763.

(4) Xi, Z.; Jiacong, S. Adv. Mater. 1999, 11, 1139-1143.

(5) Anslyn, E. V. J. Org. Chem. 2007, 72, 687-699.

(6) Dini, F.; Martinelli, E.; Pomarico, G.; Paolesse, R.; Monti, D.; Filippini, D.; D’Amico, A.; Lundström, I.; Di Natale, C. Nanotechnology 2009, 20, 055502-09.

(7) Di Natale, C.; Monti, D.; Paolesse, R. Mater. Today 2010, $13,46-52$.

(8) (a) Di Natale, C.; Paolesse, R.; D’Amico, A.; Lundström, I.; Lloyd-Spetz, A. J. Porphyrins Phthalocyanines 2009, 13, 1123-1128. (b) Paolesse, R.; Mandoj, F.; Marini, A.; Di Natale, C. Encyclopedia of Nanoscience and Nanotechnology; Nalwa, H., Ed.; American Science Publishers: Valencia, CA, 2003; Vol. 9, pp 21-43.
(9) Drain, C. M.; Varotto, A.; Radivojevic, I. Chem. Rev. 2009, 109, 1630-1658.

(10) Bussetti, G.; Cirilli, S.; Violante, A.; Chiaradia, P.; Goletti, C.; Tortora, L.; Paolesse, R.; Martinelli, E.; D’Amico, A.; Di Natale, C.; Giancane, G.; Valli, L. Appl. Phys. Lett. 2009, 95, 091906-08.

(11) Weightman, P.; Martin, D. S.; Cole, R. J.; Farrell, T. Rep. Prog. Phys. 2005, 68, 1251-1342.

(12) Adler, A. D.; Longo, F. R.; Finarelli, J. D.; Goldmacher, J.; Assour, J.; Korsakoff, L. J. Org. Chem. 1967, 32, 476.

(13) Goletti, C.; Paolesse, R.; Di Natale, C.; Bussetti, G.; Chiaradia, P.; Froiio, A.; Valli, L.; D’Amico, A. Surf. Sci. 2002, 501, 31-36.

(14) Kemp, J. C. J. Opt. Soc. Am. 1969, 59, 950-953.

(15) Goletti, C.; Paolesse, R.; Dalcanale, E.; Berzina, T.; Di Natale, C.; Bussetti, G.; Chiaradia, P.; Froiio, A.; Cristofolini, L.; Costa, M.; D'Amico, A. Langmuir 2002, 18, 6881-6886.

(16) Goletti, C.; Bussetti, G.; Chiaradia, P.; Sassella, A.; Borghesi, A. Org. Electron. 2004, 5, 73-81.

(17) Goletti, C.; Bussetti, G.; Chiaradia, P.; Sassella, A.; Borghesi, A. Appl. Phys. Lett. 2003, 83, 4146-4148. Sassella, A.; Campione, M.; Raimondo, L.; Borghesi, A.; Bussetti, G.; Cirilli, S.; Violante, A.; Goletti, C.; Chiaradia, P. Appl. Phys. Lett. 2009, 94, 073307-09.

(18) McIntyre, J. D. E.; Aspnes, D. E. Surf. Sci. 1971, 24, 417-434.

(19) Selci, S.; Ciccacci, F.; Chiarotti, G.; Chiaradia, P.; Cricenti, A. J. Vac. Sci. Technol., A 1987, 5, 327-332. In general, $A$ is related to the dispersive part of the substrate dielectric function, $B$ to the absorptive part. In a photon energy region where the substrate is not absorbing, $B$ is null. As the quartz is transparent $(B=0)$ in the whole photon energy range, the expression for $\Delta R / R$ strongly simplifies, resulting nearly coincident with the anisotropy of the absorption coefficient.

(20) Antoine parameters are available in the database of the National Institute of Standards and Technology http://webbook.nist.gov/chemistry/.

(21) De Luca, G.; Treossi, E.; Liscio, A.; Mativetsky, J. M.; Monsu Scolaro, L.; Palermo, V.; Samor, P. J. Mater. Chem. 2010, 20, 2493-2498.

(22) Gong, X.; Milic, T.; Xu, C.; Batteas, J. D.; Drain, C. M. J. Am. Chem. Soc. 2002, 124, 14290-14291.

(23) Bussetti, G.; Corradini, C.; Goletti, C.; Chiaradia, P.; Russo, M.; Paolesse, R.; Di Natale, C.; D’Amico, A.; Valli, L. Phys. Status Solidi B 2005, 242, 2714-2719.

(24) Noguez, C.; Beitia, C.; Preyss, W.; Shkrebtii, A. I.; Roy, M.; Borensztein, Y.; Del Sole, R. Phys. Rev. Lett. 1996, 76, 4923-4926.

(25) Valli, L. Adv. Colloid Interface Sci. 2005, 116, 13-44.

(26) Dunbar, A. D. F.; Brittle, S.; Richardson, T. H.; Hutchinson, J.; Hunter, C. A. J. Phys. Chem. B 2010, 114, 11697-11702.

(27) Tibuzzi, A.; Ficorella, F.; Paolesse, R.; Dalla Betta, G.-F.; Boscardin, M.; Macagnano, A.; Di Natale, C.; Soncini, G.; D’Amico, A. Sens. Actuators, B 2005, 111, 242-246.

(28) Jurs, P.; Bakken, G.; McClelland, H. Chem. Rev. 2000, 100, 2649-2678. 